\title{
PHILOSOPHICAL AND CONCEPTUAL PERSPECTIVES ON THE DESIGN OF GROUP SUPPORT SYSTEMS
}

\author{
Karma Sherif \\ and \\ James F. Courtney \\ Department of Business Analysis \& Research \\ Texas A\&M University \\ College Station, Texas USA \\ $77843-4217$ \\ (409) 845.9541 \\ kss6354@tamvm1.tamu.edu \\ J-Courtney@tamu.edu
}

\begin{abstract}
:
Design of infurmation systems (IS) has been one of the most elusive tasks of the system analyst, requiring technical cxpertisc. creattve inspiration and deep understanding of the problem under examination. Perhaps even more than is the case with other IS. group supporn systems (CSS) design is challenging hecause it involves groups who vary in behavior. process and culture. The system designer's worldview will shape its features. In an attempt to shed new light on GSS design, this paper examines the problem from four philosuphical perspectives or paradigms: Functionalism, Social Relativism, Radical Structuralism and Nechumanism. The generic requirements identified from the analysis include supporn fior multitasking. multiple languages. customization. muluple communication modes, interoperability, rational discourse and knowledge validation. While these appear to be valid requirements to suppont computer-mediated decision making, it may be difficult if nol impossible to salisfy them in one software system.
\end{abstract}

\section{INTRODUCTION}

Design of information systems has been one of the most elusive tasks of the system analyst, requiring technical expertise. creative inspiration and deep understanding of the problem under examination. It is the core of the whole endeavor of developing systems. The process of determining requirements; be it user requirements or system specifications, is very challenging. This vital role of design manifests itself everyday, especially after system implementation when it becomes evident that good design has its encouraging rewards, and that bad design is painfully expensive. Thus an incisive understanding of the problem, the social context surrounding it, and of forces interacting within, is crucial for pinpointing requirements, setting goals and envisioning change. This directed change can be predetermined by the designer and wished for in response to organizational needs or even out of social responsibility. Users can sometime require change to adapt to their environment. Whoever or whatever is driving the change, it is through design that we try to modify our environment to a better and a more purposeful one. Therefore It remains the responsibility of the designer to foresee and test the impact of the intervention on the targeted community. The worldview of the designer affects the properties of the system being designed (Churchman, 1971; Mandviwalla, 1994). And the properties of the system determine how useful it will be across various user groups and cultures. The broader the design perspective, the more likely it is that the resulting system will be suitable for heterogeneous social contexts.

Thus the objective of this paper is to provide insight into how group support systems (GSS) software, which is now being more widely deployed in global markets, can be made more suitable for a wider variety of users in various social and cultural contexts. To identify generic design requirements that would make GSS more pluralistic, we examine GSS design from broad perspectives. Since much of the anticipated outcomes of GSS use are driven by the designer's worldview, the role of the designer is examined in some detail within the context of four philosophical paradigms: Functionalism, Social Relativism, Radical Structuralism and Neohumanism (Hirschheim, et al., 1996). The paper begins by reviewing the results of studies of GSS use, and their effects on group decision outcomes and group behavior. Next GSS design is considered from the four perspectives mentioned previously. The paper ends with a proposed set of challenging generic requirements for GSS design.

\section{GROUP SUPPORT SYSTEMS (GSS)}

GSS are systems designed to improve group meetings through communication and by providing tools for structuring decision analysis (DeSanctis \& Gallupe 1987). Different features have been supported, ranging from mere communication to more complex systems allowing audio and video communication along with decision support tools ( Ayre \& Gottesman 1994). Interest in the technology stems from the belief that 
collaborative work is capable of producing performance superior to an individual's efforts due to the synergy of the group (George, Easton, Nunamaker 1990).

However certain characteristics of groups restrict the realization of their full potential or even matching the performance of its best members (Shaw 1978). Lack of coordination, diffusion of responsibility, conflict and lack of motivation (known collectively as process losses) account for limited group performance. Accordingly GSS are designed to help increase group effectiveness with regard to decision making by diminishing the effect of process losses or positively directing the characteristics to better serve the group decision outcomes.

The GSS designer's goal is to build optimal systems that will improve behavior and instruct users on techniques to better structure their problem solving effort (Feldman \& March, 1981). In accomplishing their goals, designers and developers have offered a wide variety of functions, the most common ones being :

- Structured steps for problem solving including :

Problem definition

Examination of alternatives and generation of new ideas

Decision support tools for analysis

Building consensus (Poole, Holmes \&DeSanctis 1991).

- Open communication between members.

- Providing a role for a facilitator or leader to better direct the group decision process.

\section{EFFECTS OF GSS}

Researchers have been preoccupied by the effects that technology has on groups. Reported outcomes have been inconsistent across research efforts in this subject area due to variance in group size, group structure, tasks and technology. Four main outcome areas, though, have been identified (see Table 1). These relate to processes, behavior, decision quality and organizational structure.

Process outcomes: The deterministic intervention of some GSS in the form of structured steps taken for problem solving activities identified participation, satisfaction and time to decision as process outcomes ( Easton et al 1990). GSS were found by many researchers to enhance participation among all members rather than allowing dominance by one or more participants (Hiltz, Johnson \& Turoff 1986; Nunamaker, Applegate \& Konsynski 1987; DeSanctis \& Gallupe 1987). However Beauclair (1987), Jarvenpaa, Rao \& Huber (1988) and Easton (1988) reported no effect on participation by GSS.

Inconsistent findings were also reported on time to decision. Time to decision was either found to be longer in computer mediated communication (Easton et al 1990) or unaffected (Beauclair 1987, Easton 1988).

Findings on satisfaction with the process were more inclined towards increased satisfaction for GSS groups. While a number of researcher found no effect, Gallupe, DeSanctis \& Dickson (1988) and Watson, DeSanctis \& Poole (1988) noted that satisfaction with the process and outcome was reduced by GSS.

Behavior outcome: Regarding behavior, positive outcomes such as effective conflict management and uninhibited behavior have been identified (Poole, Holmes \& DeSanctis 1991). Anonymity played an invisible role in bringing about these changes, encouraging free expression, depersonalization of discussion, rambling conflict and causing members to concentrate on issues rather than "personal antagonism" (Poole et al 1991 p934).

TABLE 1

Summary of GSS effects on groups

\begin{tabular}{|c|c|}
\hline Variables & Definition and findings \\
\hline Process outcome & $\begin{array}{c}\text { Participation, satisfaction with the process and the decision and time to } \\
\text { decision. Findings were inclined to increase participation by group members } \\
\text { and increased satisfaction with both the process and the decision. Time to } \\
\text { decision was reported longer in GSS meetings than in face to face. }\end{array}$ \\
\hline Behavior outcome & $\begin{array}{c}\text { Conflict management and uninhibited behavior. GSS empirical } \\
\text { research showed that GSS sessions reduced rambling conflict and encouraged } \\
\text { open unrestrained discussions. }\end{array}$ \\
\hline Decision Outcome & $\begin{array}{c}\text { Decision quality measured in terms of correctness, creativity and } \\
\text { uniqueness of alternatives. Experiments proved GSS sessions to result in } \\
\text { better decision quality. }\end{array}$ \\
\hline $\begin{array}{c}\text { Some decentralized organizations have centralized. Some centralized } \\
\text { Organizations have decentralized. }\end{array}$ \\
\hline
\end{tabular}


Decision outcome: Reported impacts on decision outcome include better decision quality, as measured in terms of correctness, creativity or uniqueness of alternatives (Steeb \& Johnson 1981: Lewis 1982: Turoff \& Hiltz 1982; Gallupe, DeSanctis \& Dickson 1988; Zigurs. Poole \& DeSancris 1988). Results have been relatively consistent in this area.

Organizational Design and Structure: Apart from the effects on group behavior and decision outcome, GSS is thought to have considerable impact on organizational design and structure (Huber 1990). The findings: regarding change in design and structure have also been inconsistent due to the nature of the organization and the surrounding environment. Computer-assisted communication and group decision making have led to decentralization in highly centralized organizations, and to centralization in highly decentralized organizations. Organizational structure has also undergone an ongoing change after the introduction of GSS. Through the extensive use of such systems, organizations have realized benefits of the technology and adapted their structure to better exploit these privileges. All of these contradicting results and emerging changes pose a great challenge to GSS design and makes it complicated for the designer to generalize.

\section{THE ROLE OF THE DESIGNER}

Before proceeding to the discussion of GSS design we need to comprehend the exact role that the designer plays. Is it the designer who is driving these changes in group behavior and organization structure through the implementation of the design? Or are organizations as social institutions the driving force? DeSanctis" (1993) "Individualistic" and "Collectivism" assumptions serve as a rich, but simple dichotomy for theories underlying GSS design (see Table 2).

The "individualistic" approach, or as Spacaman \& Oskamp (1990) call it "determinism", assumes that technology is capable of changing behavior. Technology is viewed as "an exogenous force which determines or strongly constrains the behavior of individuals and organizations." (Markus \& Robey, 1988, P. 585). Technology has the power to enhance individual skills and overcome deficiencies inherent in groups. Accordingly, The effectiveness of design is measured in terms of the ability of the system to help groups reach quality decisions, increase participation, reach consensus and increase satisfaction.

Deterministic changes are designed to empower the individual with skills to better serve the whole group (DeSanctis 1993). Such design is built on individuals as the unit of analysis. The challenge 10 the designer with individualistic assumptions is how to aggregate individual scores to achieve group measures. With single-user applications, design is more forward and the user has the luxury of changing the tool to match individual needs and preferences. In multi-user applications, users collaboratively use the system and are deprived of custom tailoring (Mandviwalla 1994)

TABLE 2

Assumptions underlving GSS design

\begin{tabular}{|c|c|c|}
\hline Assumption & Role of technology & \multicolumn{1}{c|}{ Implications to design } \\
\hline Individualistic & & $\begin{array}{l}\text { Systems should enhance decision } \\
\text { quality. The challenge is to achieve group } \\
\text { measures for group requirements and decide on } \\
\text { the spirit the system should adopt. }\end{array}$ \\
\hline Collectivism & deterministic & $\begin{array}{l}\text { Systems should convey society's rules } \\
\text { and values. The challenge is how to code social } \\
\text { values and rules into the system. }\end{array}$ \\
\hline
\end{tabular}

The process of translating group requirements into a system's functionality gets complicated due to the plurality of personalities and tasks, and the variety in a group context. The question then arises as to which requirements should the system serve? What worldview should the designer instill upon the system? The worldview being the inner beliefs that one holds about the world and that unconsciously influences what one does and the way one chooses to do it (Whiteside \& Wixon 1988). Accordingly designers rely on explicit or implicit worldviews to guide their design and development effort. Understanding the worldview of a designer is crucial to understanding the system's belief structure and verifying its technical features. The worldview of a system is one aspect of the technological structure viewed as the objectives in terms of goals and attitudes that the technology wishes to accomplish.

Poole \& DeSanctis (1990) have clearly distinguished between the "spirit" and the "structural features" of a system in their proposed "Adaptive Structuration Theory." The "spirit" is defined as the worldview and the "structural features" as the translated design issues that serve the "spirit." Thus the "structural features" are 
functionally dependent on the system's "spirit." Should the system"s "spirit" then reflect the worldview of the designer? That is, what the designer regards appropriate and socially acceptable. Or should it convey the beliefs of a group leader and serve the motives of the elite? Can the designer be objective and detach his or her own views from the design process to support the worldviews of the group and satisfy their requirements? Can a "Utopian" system attending equally to the needs of all groups and all members be built? How can the designer accommodate diversities in race, gender and culture in GSS design?

Collectivism views organizations as social structure, and technology as an outcome of the social culture (DeSanctis 1993). Such an assumption might help explain the contradictory findings regarding the impact that technology brings on groups and organizations. Since culture changes, technology will have different meanings, different roles and different purposes across organizations and groups and along time. It is these social complexities that affect technology development. The designer's goal is seen as a way to reinforce cultural rules and values, to encode cultural norms into GSS, and "to bring gradual improvement in the social order of the organization or community" (DeSanctis 1993 p.98).

According to collectivism the world view of the system would convey the rules and values of the organization. The concern is more about the goals society is trying to achieve through the technology rather than the goals of the system. The impact GSS is thought to have on groups and organizations is considered to be a function of the surrounding social context. Designers must be socially aware of users' needs and cultural values instead of being consumed by the technology. Such social concern will help minimize wasted resources deployed on the development of fancy eye-catching tools that are not fulfilling their social obligation. The challenge posed to the designer is how to code social values and rules into the system? How can the system reflect society's values and norms to assertively alter the community in a productive way?

In trying to determine answers for these inquiries a leading step would be to examine the different philosophical paradigms underlying the design of current GSS. A subsequent step would be to explore group requirements and the challenges they proclaim to the designer.

\section{PARADIGMATIC FOUNDATIONS OF IS AND THEIR APPLICATION TO GSS}

Hirschheim, Lyytinen \& Klein (1996) classify major theoretical assumptions underlying IS design under four philosophical paradigms: Functionalism, Social Relativism, Radical Structuralism and Neohumanism. These are summarized in Table 3.

Functionalism is pursuing the design of systems that have specific and clear objectives. It should serve the decision maker by providing timely and meaningful information to help solve organizational problems. According to this approach management is the best candidate for providing the specific and clear goals of a system. The designer acts as a technology expert interpreting system objectives into a structured formalized set of steps and procedures. This approach assumes that there is only one face to reality which is modeled in a purely technical fashion. Change is deterministic in nature and predetermined by management and the designer. The designers comprehension (possibly affected by his or her own views) of management's needs are reflected in the system's features and its "spirit."

Aspects of Functionalism do apply to GSS design. Most of the current GSS systems offer tools for the formalization and structuring of problem solving procedures. As mentioned earlier, positive outcomes were reported regarding decision quality that were believed to be the results of imposing structuration. Most of these systems were built on scientific management theories. It does however, consider social conventions that, to a great extent, determine the outcome.

TABLE 3

ISD Paradigms and their application to GSS

\begin{tabular}{|c|c|c|}
\hline Paradigm & Emphasis on & $\begin{array}{c}\text { GSS features that support } \\
\text { the paradigm }\end{array}$ \\
\hline Functionalism & $\begin{array}{c}\text { Technical rationality and } \\
\text { legitimate management needs }\end{array}$ & $\begin{array}{c}\text { Structuring and formalization } \\
\text { of problem solving techniques }\end{array}$ \\
\hline Social relativism & $\begin{array}{c}\text { Sense making and mutual } \\
\text { understanding }\end{array}$ & $\begin{array}{c}\text { Encouragement } \\
\text { participation and building consensus }\end{array}$ \\
\hline $\begin{array}{c}\text { Radical } \\
\text { Structuralism }\end{array}$ & Elass interest of the working & Anonymous communication \\
\hline Neohumanism & Emancipation & Not yet applied \\
\hline
\end{tabular}


But Functionalism fails to explain why conflicting results have been reported. It can be inferred that GSS systems that are not targeted to a specific group tend to support Functionalistic views, anticipating similar outcomes across groups and assuming that rules and patterns govern the reality that we perceive. GSS systems that concentrate on diminishing the effect of process losses through structuring apply Functionalism. Technology is believed to cope successfully with problems associated with group work regardless of human behavior (Mandviwalla 1994).

The central flaw of this approach is inadequate understanding of meaning in the construction of reality. Functionalism forgets that "the link between designs and the effects they produce is the meaning attributed to them" (Hirschheim, et al. 1996. p.108).

Social Relativism, the second paradigm, concentrates on building systems that encourage sensemaking and mutual understanding. There is no one accepted reality for what we discem or what we anticipate. Perceptions is what we have and perceptions vary depending on the environment, previous experiences and culture. The designer's role is to ease the transition from one perception to the other through continuous interaction.

GSS system: that promote information exchange among group members apply Social Relativism. Through interaction, reality is socially constructed. Technology is viewed as a tool for addressing problems through participation. Building consensus via group acceptance is of major concern. Right or wrong is relative and what counts is what the group agrees on and perceives as emotionally and morally right. Functionalists might argue that social relativism would result in emotion-based, effectively questionable decisions. However, research in cognitive science has indicated that nonrational "socioemotional" decisions often turn to be highly functional (Etzioni 1988).

Radical structuralism is mainly concerned with building systems that promote the interests of the working class. rather than limiting the design focus to management. This paradigm strongly urges the designer to advocate the labor side and to adjust the balance of power between management and labor. To some extent, this insight is captured in GSS design and supported by anonymous communication. Subordinates are given the right to freely express their views and to strongly defend their beliefs against management. Though GSS systems encourage participation, the power bestowed on workers is still limited to their competency and their ability of persuasion. The problem with these systems is their deliberation of conflict as a vital sign for group discussions, thereby adding to the complexity of the decision process.

The Neohumanist approach has taken from all three aforementioned paradigms and added the notion of "emancipation." Emancipation is defined as the ability of information systems to "facilitate the widest possible debate of organizational problems such that truly shared objectives could be agreed upon as well as policies for achieving them" (Hirschheim, et al., 1996, p.87). The GSS system designer should try to balance between technical intervention, shared understanding and "continued emancipation." The focus should be on freeing groups and organizations from distoring influences and other constraints to "rational discourse."

Neohumanism is considered a sound theory, however, no work has been reported that fully supports it. Its idealistic nature assumes that "rational discourse" can be attained without suggesting how. This has led to the reluctance of designers to apply the concept. It is the application of Neohumanism to GSS design that forms the general challenge since it considers all aspects be it technical, social or emancipatory.

\section{IMPLICATIONS FOR GSS DESIGN}

GSS sofiware presently reflecis a design based primarily on functionalistic assumptions. By being cognizant of these assumptions, and reflecting upon the implications of the other paradigms, hopefully will result in a set of GSS design requirements that are more neohumanistic in nature. Of course, no design can satisfy all the requirements for all groups for all time. Nevertheless, by starting from a broader perspective, it seems reasonable to expect to produce a more pluralistic set of generic requirements than has been the case with previous efforts (see Figure 1). Such a set of requirements is described in Table 4. 


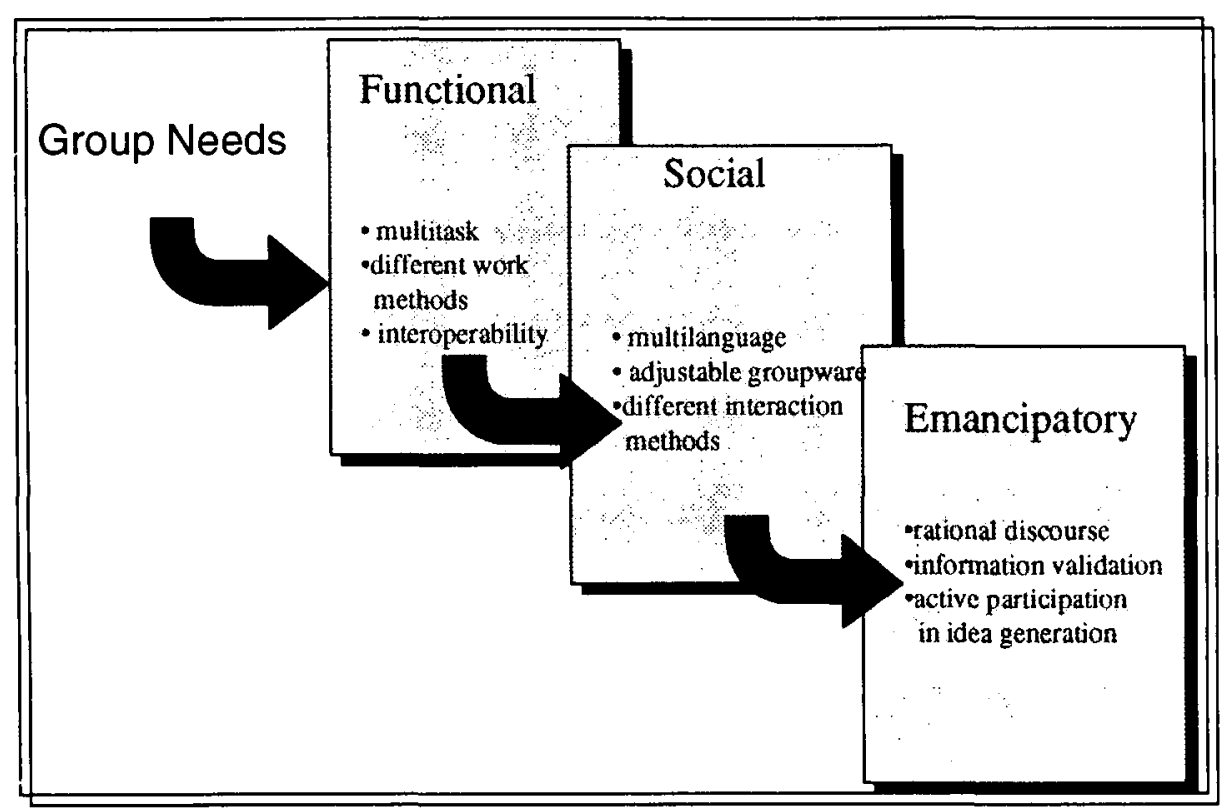

FIGURE 1. Group requirements

First, within a single group, understanding the dynamics of group characteristics, group process and decision outcomes is multidisciplinary. We need to take a wider view of group work studying "Organizational Theory, Management Science, Social Psychology, Decision Theory and Communication" (Mandviwalla 1994 p.249). There are multiple tasks involved in the decision making process and a variety of work methods for each task. Groups differ in the tasks they choose and the way they perform them.

TABLE 4

Summary of GSS Requirements

\begin{tabular}{|c|c|}
\hline GSS Feature & Description \\
\hline Multitasking & Support for multiple tasks in the decision-making process. \\
\hline Leadership style & $\begin{array}{c}\text { Support worldviews reflecting different leadership styles and user } \\
\text { participation modes. }\end{array}$ \\
\hline Multilinguistic & Support for multiple languages. \\
\hline Multcommunication & Support for text, audio and video communications modes. \\
\hline Customization & Tailorable to the needs of the group. \\
\hline Interoperability & Seamless integration with other popular software packages. \\
\hline Rational discourse & Support for Robert's rules of order and structured debates. \\
\hline $\begin{array}{c}\text { Knowledge } \\
\text { validation }\end{array}$ & $\begin{array}{c}\text { Provide means to validate and manage information and knowledge } \\
\text { brought into the decision group process. }\end{array}$ \\
\hline
\end{tabular}

Second the conflict on the system worldview must be recognized and accommodated. The choice of a worldview should be carefully and objectively rendered to fit with the organizational culture and to accord with the social meaning. Group culture must also be considered. Though Aiken, Hwang, De Magalhaes \& Martin (1993) had reported no significant differences between cross cultural groups in terms of ease of communication, fear of peer criticism and overall meeting satisfaction, special consideration should be given to 
issues such as race, gender and beliefs. For example leadership and participation is viewed differently across culture. It might be true that groups in US. organizations value participation and appreciate the point view of each and every member. It is to be expected then. that these groups would oppose deterministic intervention of a leader with the decision process. Their acceptability of products with limited selections would be low.

However, it is highly questionable that the same will hold with Middle Eastern groups. The leader in a country such as Egypt is the catalyst who promotes productivity by managing people and the decision process. A more authoritarian approach may be acceptable in such cultural contexts.

As organizations become more global in nature, GSS are being used in wider and more varied linguistic domains. It is important to consider language in future GSS, as mono-linguistic groups may no longer be considered the sole users of such systems.

Religion may also be decisive factor in group acceptance of a GSS in some cultures. In cultures where religion plays a major social and political role, it would be naive to ignore its implications on the group's implicit needs or approval of a product. A considerable sector of Egyptians, for instance. are highly swayed by religion, and systems that are compatible with religious beliefs would easily appeal to their personalities. There is only one reality beyond any shadow of doubt; that defined by the Holy Book. This fact was contirmed when Islamic investments companies were launched in 1980's and were a huge success signaling an alarm to government of a massive takeover. The designer's understanding of the relativity of "universal truths" and the uniqueness of groups should be reflected in the design (Mandviwalla \& Olmen 1994)

In order to ensure group satisfaction and a high utilization rate, the system should support multi-group tasks through different system modules (Mandviwalla \& Olmen 1994, Huber 1984). The broadening of the system's functionality certainly will defy the designer unless access to various other software products from different vendors are incorporated into the design.

Besides multiple tasks, systems should support multiple work methods for each task to serve the needs of different groups (Hackman 1990). The freedom of choice is more cherished than a predetermined fixed set of functions (Malone \& Lai 1992). For example, Dykstra \& Carasik (1991) present a "conversation-based" design that offers the group the freedom to establish its structure.

Groups also require multiple interaction methods which they use interchangeably to accommodate to changes in the criticality of the job. A GSS should provide both face to face communication through audio and video media. The whole concept is creating adjustable groupware that can be easily customized by the group to better serve its needs.

Accordingly, designers need to consider possibilities of the system migration across applications modelessly. Groups often require information saved on different software or they might occasionally need to conduct professional analysis provided by a separate tool.

In certain contexts, such as formal legislative bodies and the like, it may be useful for the system to impose restrictions on group communication, thereby leading to a form of "rational discourse." As DeSanctis and Gallupe(1987) have suggested, it might be helpful to enforce Robert's rules of order. Or. Hodges' dialectical engine (1991) might be incorporated into negotiation systems to enforce rules of structured debate (Rescher. 1977).

A final and highly neglected aspect of collaborative work that designers should consider is the ability of the system to validate input brought into by group members. According to Hirschheim. Lyytinen and Klein (1996) IS is defined as "technically mediated social interaction systems aimed at creating, sharing and interpreting a wide variety of meaning." In that respect GSS falls short of creating meaning and is limited in interpreting it. Validation of information is vital because of the influential power that information exerts on individuals. A GSS system should be able to endorse or falsify ideas and proposals initiated by group members. A GSS that can extrapolate and fill in unspecified gaps can be greatly helpful.

For example, Liou \& Nunamaker (1993) propose a GSS that facilitates knowledge acquisition from multiple experts to build an information center help-service expert system. The electronic documentation of experts' knowledge. the structured process, the parallel knowledge extraction and the collaborative atmosphere enriched the domain of expertise. A definite challenge would be to take this research a step forward and incorporate the expert system knowledge base within GSS to help groups generate ideas and facilitate discussion. Earlier discussions on particular problems can be searched and the effectiveness of the decision outcome can be evaluated against the results. Qualified decisions can be incorporated into the knowledge base as a learning experience.

The design of an active participating GSS is definitely difficult. It is hard to agree on standardized labels for everything around us. The need to standardize our description of specific experiences and agree on common names for simple labels is a compelling prerequisite for the design of a "Lockean system" (Churchman 1971). Another challenge is the community agreement for what is true or false and a willingness to accept the 
worldviews of professional community members. The problem however, is the system's presumption that the future will follow the lead of the present in an "isomorphic sense" or else will be unreliable.

\section{CONCLUSION}

Design is the pillar for the development of highly operable and productive systems. The goal of a GSS is improving group decision processes and outcomes by providing communication and techniques for supporting group endeavors. The design of a GSS is complicated because it involves groups whose behavior and decision process are hard to predict. Therefore it is crucial to understand the group before attempting to determine its requirements and choosing a system worldview. The major assumptions and paradigms underlying GSS design were examined as a starting point In determining group requirements. A generic set of requirements for GSS systems groups were proposed. Though not all of the requirements can be modeled they certainly present valid needs for groups to effectively and efficiently proceed with computer mediated decision making.

\section{REFERENCES}

Aiken, M.,C. Hwang, R. De Magalhaes,\& J. Martin (1993). A comparison of Malaysian and American groups using a group decision support system. Journal of Information Science Principles \& Practice. 19(6) 489-491.

Ayre, R., \& Gottesman, B.Z. (1994). Workgroup Computing Group Enabled. PC Magazine 13(11), 171189.

Beauclair, R. A. (1987). An experimental study of the effects of group decision support system process control applications on small group decision making, unpublished $\mathrm{Ph}$. D. dissertation, Indiana University, Bloomington, IN, 1987.

Churchman, C.W. (1971) The design of Inquiring Systems : Basic concepts of systems and organization Basic Books, Inc., NY.

DeSanctis, G. (1993) Group support systems new perspectives Shifting foundations in group support system research. Macmillan , NY.

DeSanctis, G., \& Gallupe, R. B. (1987). A Foundation for the study of group decision support systems. Management Science, 33(5), 589-609.

Dykstra, E. \& Carasik, R. 1991. Structure and support in cooperative environments: The Amsterdam Conversation Environment. Int. J. Man-Mach. Stud. 34, 419-434.

Easton, G. K., (1988). Group decision support systems vs. face-to-face communication for collaborative group work: an experimental investigation. Doctoral dissertation, University of Arizona .

Etzioni , A. (1988). The moral dimension: toward a new economics. New York: The Free Press.

Feldman, M. S., \& March, J. G. (1981). Information in organizations as signal and symbol. Administrative Science Quarterly, 26(2), 171-186.

Gallupe, R. B., G. DeSanctis, \& G.W. Dickson, (1988). Computer based support for group problemfinding: an experimental investigation, MIS Quarterly., 12(2), 277-296.

George, J. F., Easton, g. k., Nunamaker, J. F., Jr. Northcraft, G. B. (1990). A study of collaborative group work with and without computer-based support. Information Systems Research, 1(4), 394415.

Hackman, J. R. (1990) Groups that work and those that don't. Jossey-Bass, San Francisco.

Hiltz, S. R., Turoff, M., \& Johnson, K. (1989). Experiments in Group decision making,3: Disinhibition, deindividuation, and group process in pen name and real name computer conferences. Decision Support Systems, 5(2), 217-232.

Hodges, W. S. (1991). DIALECTRON: A Dialectical Engine for the Support of Strategic Planning. Unpublished Ph. D. dissertation, Texas A\&M University.

Hirschheim, R., Lyytinen, K. \& Klein, H. (1996) Information system development and data modeling : conceptual \& philosophical foundations, Cambridge University Press.

Huber, G. P. (1984) Issues in the design of group decision support systems. MIS Quart. 3, 195-204.

Huber, G. P. (1990). A theory of the effects of advanced information technologies on organizational design, intelligence, and decision making. Academy of Management Review, 15(1), 47-71.

Jarvenpaa, S. L., V. S. Rao, \& Huber G. P., (1988). Computer support for meetings of group working on unstructured problems: A field experiment, MIS Quart ., 12(4) ,645-666.

Lewis, F. L.. (1982). Facilitator: a micro computer decision support systems for small groups. Doctoral dissertation, University of Louisville, 1982 
Liou, Y. I. \& Nunamaker, J. F. Jr. (1993) An investigation into knowledge acquisition using a group decision support system. Information \& Management. 24(3) 121-132.

Malone, T. and Lai, K (1992) Toward intelligent tool for information sharing and collaboration. In Computer Augmented Teamwork, R. Bostrom, R. Watson and S. Kinney, Eds. Van Nostrand Reinhold, New York, 86-107.

Mandviwalla, m. \& Olfman, Lorne (1994). ACM Transactions on Computer-Human Interaction, 1(3), 245-268.

Mandviwalla, M., (1994). The world view of collaborative tools. The Arachnet electronic journal on virtual culture, 2(2) 1994.

Markus, M. L., \& Robey, D. Information technology and organizational change: Casual structure in theory and research. Management Science, 1988, 34(5), 583-598.

Nunamaker, J. F., Applegate, L. M., \& Konsynski, B. R. (1988). Computer-aided deliberation: model management and group decision support. Operations Research, Special issue on Decision Support Systems, 36(6),826-848.

Poole, M. S., Holmes, M., \& DeSanctis, G. (1991). Conflict management in a computer-supported meeting environment. Management Science, 37(8), 926-953.

Rescher, N. (1977). Dialectics: A Controversy-Oriented Approach to the Theory of Knowledge, State University of New York press, New York.

Steeb, R. \& S. C. Johnson, (1981) A computer-based Interactive system for group decisionmaking. IEEE transactions on systems, man and cybernetics, SMC-11,8, 544-552

Turoff, M. \& S. R. Hiltz. (1982). Computer support for group versus Individual decisions. IEEE Transactions on communications, 30(1), 82-91.

Watson, R. T., G. DeSanctis, \& M. S. Poole, (1988). Using a GDSS to facilitate group consensus: some intended and unintended consequences, MIS Quart., 12(3), 463-478.

Whiteside, J \& Wixon, D. (1988) Contextualism as a world view for the reformation of meetings. In CSCW Proceedings. ACM, New York, 369-376.

Zigurs, I., M. S. Poole, \& G. DeSanctis, A study of influence in computer-mediated group decision making, MIS Quart., 12(4), 625-644. 